\title{
ANÁLISE DE AGRUPAMENTO DE CARACTERES MICROSCÓPICOS DO LENHO DE ESPÉCIES SUL-BRASILEIRAS DE MIMOSA L. ${ }^{1}$
}

\author{
PAULO FERNANDO DOS SANTOS MACHADO² JOSÉ NEWTON CARDOSO MARCHIORI ${ }^{3}$
}

\begin{abstract}
RESUMO
A análise de agrupamento de caracteres anatômicos envolveu lenhos de vinte e duas espécies sul-brasileiras de Mimosa L. (Fabaceae: Caesalpinioideae). Para o estudo, selecionaram-se quatorze caracteres anatômicos, com vistas ao reconhecimento de grupos infragenéricos. Confirmou-se a heterogeneidade estrutural referida na literatura. A ocorrência de caracteres primitivos no lenho recomenda um posicionamento basal do gênero no clado Mimosoideae e subfamília Caesalpinioideae. A série Stipellares, representada por M. barnebiana, M. bifurca, M. cruenta, M. intricata, $M$. trachycarpa e M. uraguensis, compõe um grupo distinto, pelos raios homogêneos e poros racemiformes, com arranjo tendente a dendrítico. Tanto na seção Calothamnos (M. berroi, $M$. daleoides, M. eriocarpa, M. flocculosa, M. incana, M. pilulifera, M. scabrella e M. taimbensis) como na subsérie Obstrigosae (M. adpressa, M. parvipinna, M. ramulosa), os caracteres diagnósticos carecem valor taxonômico reconhecido, distinguindo-se apenas por caracteres quantitativos. O reduzido número de espécies investigadas nas séries Bimucronatae (M. bimucronata), Habbasia (M. pigra) e Myriophyllae (M. balduinii, M. micropteris), bem como na subsérie Sparsae (M. sparsa), não permite inferências taxonômicas.

Palavras-chave: Anatomia da madeira; Caesalpinioideae; Calothamnos; Clado Mimosoideae; Fabaceae; Mimosa; Obstrigosae; Stipellares.
\end{abstract}

\section{ABSTRACT}

[Cluster analysis of microscopic wood features from South-Brazilian species of Mimosa L.].

The cluster analysis of anatomical features involved woods from twenty-two South-Brazilian species of Mimosa L. (Fabaceae: Caesalpinioideae). Fourteen anatomical characters were selected for study, with the aim to recognizing infrageneric groups. The structural heterogeneity reported in the literature was confirmed. The occurrence of primitive characters in the wood recommends a basal positioning of the genus in Clado Mimosoideae and Subfamily Caesalpinioideae. The Stipellares series, represented by M. barnebiana, M. bifurca, M. cruenta, M. intricata, M. trachycarpa and M. uraguensis, make up a distinct group, consisting of homogeneous rays and racemiform pores, with dendritic arrangement. Both in section Calothamnos (M. berroi, $M$. daleoides, M. eriocarpa, . flocculosa, . incana, M. pilulifera, $M$. scabrella and M. taimbensis) as in subseries Obstrigosae (M. adpressa, M. parvipinna, M. ramulosa), the selected characters lack recognized taxonomic value, being species distinguished only by quantitative features. The small number of species investigated in the Bimucronatae series (M. bimucronata), Habbasia series (M. pigra), Myriophyllae series (M. balduinii, M. micropteris), as well as in the Sparsae subseries (M. sparsa), does not allow taxonomic inferences.

Keywords: Caesalpinioideae; Calothamnos; Clado Mimosoideae; Cluster Analysis; Fabaceae; Mimosa; Obstrigosae; Stipellares; Wood Anatomy.

1 Recebido em 21-09-2019 e aceito para publicação em 29-10-2019.

2 Engenheiro Florestal, MSc., doutorando do Programa de Pós-Graduação em Engenharia Florestal, Universidade Federal de Santa Maria. barcasole@gmail.com

3 Engenheiro Florestal, Dr. Professor Titular aposentado da Universidade Federal de Santa Maria. marchioricfl@gmail.com

\section{INTRODUÇÃO}

Apesar do grande número de espécies de $M i$ mosa L. (Fabaceae: Caesalpinioideae) existentes no Sul do Brasil, a anatomia do lenho nesse gênero botânico ainda é pouco investigada, sobretudo no tocante a táxones infragenéricos. Com mais de noventa binômios válidos na flora 
regional - e espécies que variam de ervas a árvores de porte médio -, restringiu-se a presente análise de agrupamento a caracteres microscópicos do lenho de vinte e duas espécies, a partir de dados colhidos da literatura.

\section{REVISÃO DE LITERATURA}

A taxonomia do gênero Mimosa baseia-se nos estudos magistrais de George Bentham e Rupert Barneby.

Bentham (1875) organizou as espécies brasileiras de Mimosa em duas seções - Eumimosa DC. e Habbasia Benth. -, distintas entre si pela presença de flores haplostêmones e diplostêmones, respectivamente. Para Eumimosa, este leguminólogo reconheceu 12 séries: Sensitivae, Modestae, Castae, Pectinatae, Pudicae, Hirsutae, Pedunculosae, Meticulosae, Myriophyllae, Spiciflorae, Obstrigosae e Lepidotae. No caso das Habbasia, o autor segregou as espécies em nove séries: Leptostachyae, Glanduliferae, Rubicaules, Stipellares, Leptopodae, Somnianthes, Asperatae, Adversae e Pachycarpae. De larga vigência, esse esquema predominou na literatura taxonômica por mais de cem anos.

A partir de ancestrais Piptadenióides, Barneby (1991) reconheceu cinco seções em Mimosa L.: Mimadenia é a primeira do esquema, seguida por Batocaulon, que deu origem às seções Calothamnos e Habbasia; a partir dessa última, por fim, é que teria derivado a seção Mimosa L.

Com exceção de Calothamnos, que não apresenta subdivisões e tem a abrangência da série Lepidotae Benth., as demais seções de Barneby (1991) compreendem várias séries e/ou subséries. A posição das espécies investigadas em ambos os esquemas é apresentada na Tabela 1.

Pelo número de espécies investigadas destacam-se no presente estudo: a série Stipellares Benth.; a subsérie Obstrigosae (Benth.) Barneby; e a seção Calothamnos Barneby.

Vinculada à seção Batocaulon DC., a série Stipellares foi reconhecida com base nos seguin- tes aspectos morfológicos: arbustos inermes ou providos de acúleos esparsos; capítulos globosos axilares; legumes sub-planos de valvas indivisas; estipelas orbiculares ou ovais; e folíolos pouco abundantes. Para o grupo, Bentham (1875) reconheceu oito espécies; Barneby (1991) cita dezenove para a mesma, indicando, como área de distribuição, o Uruguai, Argentina, Paraguai, sul do Brasil (até São Paulo) e encosta leste dos Andes, na Bolívia. Para Santa Catarina, Burkart (1979) relaciona Mimosa hassleriana (atual $M$. bifurca), $M$. trachycarpa e $M$. cruenta; além destas, Rambo (1966) menciona para o Rio Grande do Sul: Mimosa amphigena, M. bracteolaris, $M$. intricata e $M$. iperoensis, número ampliado por pesquisas de campo mais recentes.

Segundo Barneby (1991), a subsérie Obstrigosae reúne dez espécies de arbustos e subarbustos lenhosos que são facilmente reconhecidos pelas setas adpressas (dirigidas para a base) em ramos e pedúnculos, bem como pelas folhas unijugas e capítulos globosos axilares ou em racemos. Geralmente aculeadas, existem espécies inermes (Burkart, 1979) e, salvo Mimosa tandilensis e M. ourobrancoensis, as demais são nativas no Rio Grande do Sul (Barneby, 1991).

Com a mesma abrangência da série Lepidotae sensu Bentham, a seção Calothamnos Barneby reúne árvores, arbustos e subarbustos com abundante indumento de pelos multicelulares arborescentes ou estrelados em ramos jovens, folhas e estruturas reprodutivas, bem como pelas flores funcionalmente haplostêmones e, como regra, tetrâmeras. Trata-se do grupo infragenérico mais numeroso na flora sul-brasileira.

Das espécies investigadas, salientam-se, ainda: a série Bimucronatae Barneby (seção Batocaulon DC.), por incluir o maricá (Mimosa bimucronata); a série Habbasia (DC.) Barneby (seção Habbasia DC.), à qual pertence M. pigra; a série Myriophyllae Benth. (seção Mimosa L.), por incluir M. balduinii e $M$. 
TABELA 1 - Posição taxonômica das espécies investigadas, segundo os esquemas de George Bentham e Rupert Charles Barneby.

\begin{tabular}{|c|c|c|c|c|c|}
\hline \multirow[b]{2}{*}{ Espécies } & \multicolumn{2}{|c|}{ Bentham (1875) } & \multicolumn{3}{|c|}{ Barneby (1991) } \\
\hline & Seção & Série & Seção & Série & Subsérie \\
\hline M. adpressa & Eumimosa & Obstrigosae & Mimosa & Mimosa & Obstrigosae \\
\hline M. balduinii & Eumimosa & Myriophyllae & Mimosa & Myriophyllae & \\
\hline M. barnebiana & Habbasia & Stipellares & Batocaulon & Stipellares & \\
\hline M. berroi & Eumimosa & Lepidotae & Calothamnos & & \\
\hline M. bifurca & Habbasia & Stipellares & Batocaulon & Stipellares & \\
\hline M.bimucronata & Habbasia & Rubicaules & Batocaulon & Bimucronatae & \\
\hline M. cruenta & Habbasia & Stipellares & Batocaulon & Stipellares & \\
\hline M. daleoides & Eumimosa & Lepidotae & Calothamnos & & \\
\hline M. eriocarpa & Eumimosa & Lepidotae & Calothamnos & & \\
\hline M. flocculosa & Eumimosa & Lepidotae & Calothamnos & & \\
\hline M. incana & Eumimosa & Lepidotae & Calothammos & & \\
\hline M. intricata & Habbasia & Stipellares & Batocaulon & Stipellares & \\
\hline M. micropteris & Eumimosa & Myriophyllae & Mimosa & Myriophyllae & \\
\hline M. parvipinna & Eumimosa & Obstrigosae & Mimosa & Mimosa & Obstrigosae \\
\hline M. pigra & Habbasia & Asperatae & Habbasia & Habbasia & \\
\hline M. pilulifera & Eumimosa & Lepidotae & Calothamnos & & \\
\hline M. ramulosa & Eumimosa & Obstrigosae & Mimosa & Mimosa & Obstrigosae \\
\hline M. scabrella & Eumimosa & Lepidotae & Calothamnos & & \\
\hline M. sparsa & Eumimosa & Obstrigosae & Mimosa & Mimosa & Sparsae \\
\hline M. taimbensis & Eumimosa & Lepidotae & Calothamnos & & \\
\hline M. trachycarpa & Habbasia & Stipellares & Batocaulon & Stipellares & \\
\hline M. uraguensis & Habbasia & Stipellares & Batocaulon & Stipellares & \\
\hline
\end{tabular}

Fonte: Autores. 
micropteris; e a subsérie Sparsae Barneby (da série Mimosa L. e seção Mimosa L.), pelo estudo de M. sparsa.

Uma das primeiras referências anatômicas ao gênero deve-se a Record \& Hess (1949), autores que, baseados em Mimosa scabrella e $M$. schomburgkii, não fazem qualquer menção à estrutura microscópica do lenho, limitando-se a caracteres gerais e organolépticos. Metcalfe $\&$ Chalk (1972), por sua vez, nem referem o gênero em sua extensa diagnose anatômica de Mimosaceae.

O estudo mais abrangente sobre o lenho em Mimosa L. data dos anos cinqüenta do século passado e foi realizado por Cozzo (1951), autor que ressalta a ampla variação estrutural no gênero e a ausência de característica peculiar, comum ao conjunto de espécies. Embora ainda pouco investigado, devido ao escasso interesse econômico e ao pequeno porte da maioria das espécies, cabe salientar que a variabilidade referida pelo autor argentino corresponde ao descrito para toda a subfamília Mimosoideae.

Na chave para treze espécies, Cozzo (1951) refere os seguintes caracteres anatômicos: anéis de crescimento escassamente definidos, melhor demarcados em M. ostenii; porosidade difusa, tendente a circular em M. ostenii; arranjo dendrítico "bem notável" em $M$. hassleriana e M. uliginosa, com tendência a esse arranjo em $M$. cruenta e $M$. uraguensis; vasos solitários e em múltiplos de 2-3, menos comumente 4-5, mas com longos múltiplos nas espécies de porosidade dendrítica; vasos de seção oval, angulares e de paredes delgadas em $M$. pigra, espessas em $M$. polycarpa; elementos vasculares retilíneos ou levemente sinuosos; placas de perfuração simples, oblíquas; apêndices ausentes ou curtos, por vezes longos; pontoações intervasculares ornamentadas, pequenas a médias, alternas e quase sempre próximas entre si, embora não coalescentes; pontoações raiovasculares e parênquimo-vasculares, semelhantes às intervasculares; tilos e espessamentos espiralados, geralmente ausentes; espessamentos espiralados, em elementos muito curtos de $M$. cruenta; traqueóides vasculares escassos, em espécies de porosidade dendrítica; raios heterogêneos a quase homogêneos, exclusivamente unisseriados em $M$. pigra, predominantemente unisseriados em $M$. uraguensis, geralmente trisseriados em $M$. adpressa, e com multisseriados estreitos (até 5 células) em $M$. bimucronata e $M$. polycarpa; raios de até 50 células de altura (80 em $M$. adpressa), com células envolventes curtas, em poucas espécies, e células radiais oleosas em $M$. bimucronata; parênquima axial abundante em $M$. cruenta, $M$. detinens e $M$. hassleriana, menos desenvolvido em M. adpressa, M. bimucronata e M. scabrella; células parenquimáticas axiais em séries e fusiformes, com predomínio de fusiformes em M. pigra; fibras libriformes muito curtas, de paredes finas a semi-espessas, frequentemente septadas e com fibrotraqueóides em $M$. polycarpa (Cozzo, 1951); e estratificação incompleta, embora uniforme, em M. adpressa e M. scabrella (Cozzo, 1949).

\section{MATERIAL E MÉTODOS}

As vinte e duas espécies investigadas são relacionadas a seguir, indicando-se, entre parênteses, a fonte da qual foram extraídos os dados qualitativos e quantitativos utilizados na análise de agrupamento: Mimosa adpressa Benth. (Machado \& Marchiori, 2017c); M. balduinii Burkart (Machado \& Marchiori, 2017b); $M$. barnebiana Fortunato \& Tressens (Machado et al., 2016b); M. berroi Burkart (Marchiori, 1996a); M. bifurca Benth. (Machado et al., 2016b); M. bimucronata (DC.) O. Kuntze (Marchiori, 1993); M. cruenta Benth. (Marchiori, 1985); M. daleoides Benth. (Marchiori, 1982); M. eriocarpa Benth. (Carnieletto \& Marchiori, 1993); M. flocculosa Burkart (Pereira et al., 2013); M. incana Benth. (Marchiori, 1996c); M. intricata Benth. (Machado \& Marchiori, 2017a); M. micropteris Benth. (Siegloch et al., 2013); M. parvipinna Benth. (Machado \& Marchiori, 2016a); M. pigra L. (Machado \& Marchiori, 2016b); M. pilulifera Benth. (Marchiori \& Muñiz, 1997a); M. 
ramulosa Benth. (Machado \& Marchiori, 2016a); M. scabrella Benth. (Marchiori, 1995); M. sparsa Benth. (Maccari \& Marchiori, 1994); M. taimbensis Burkart (Machado \& Marchiori, 2017a); M. trachycarpa Benth. (Marchiori, 1997b); e M. uraguensis Hook. \& Arn. (Marchiori 1996b).

Para a análise de agrupamento, selecionaramse quatorze caracteres anatômicos apontados pela literatura como promissores ao reconhecimento de eventuais grupos infragenéricos: placas de perfuração simples; pontoações raiovasculares semelhantes às intervasculares; raios heterogêneos; raios homogêneos; poros, sobretudo solitários e em múltiplos radiais; poros, sobretudo em múltiplos racemiformes; estratificação parcial; raios com até duas células de largura; raios com até três células de largura; raios com até quatro células de largura; raios com mais de quatro células de largura; raios com até $700 \mu \mathrm{m}$ de altura; raios com altura de 700 a $1.400 \mu \mathrm{m}$; e raios com mais de 1.400 $\mu \mathrm{m}$ de altura. Para cada uma das espécies investigadas, a presença ou ausência destes caracteres anatômicos é apontada, na Tabela 2, pelos dígitos "1" ou "0", respectivamente.

$\mathrm{Na}$ análise de agrupamento propriamente dita, elaborou-se uma matriz de distância de dissimilaridade de Jaccard, utilizando-se, para a geração dos grupos, o método de peso proporcional (Unweighted Pair-Group Method using Arithmetic Averages - UPGMA). Os dados foram processados no software $\mathrm{R}$, pacote Vegan (Oksanen et al., 2012). A validação do dendrograma foi calculada pelo coeficiente de correlação cofenético ( $r$ de Pearson).

\section{RESULTADOS}

O dendrograma da Figura 1 demonstra a maior ou menor proximidade entre as espécies investigadas, sendo que a estimativa da medida de similaridade ou distância entre as mesmas é representada pelo coeficiente de correlação cofenético. $O$ valor encontrado $(r=0,85)$ indica a proximidade entre as espécies, ou seja, o grau de semelhança ou diferença entre as mesmas.

A distância entre os grupos de espécies ficou em torno de 0,7 , sendo que, na maior parte, as mesmas se agruparam com 0,4 de dissimilaridade, valores tidos como baixos e que demonstram a semelhança (ou proximidade) das espécies e homogeneidade nos agrupamentos.

A leitura do dendrograma é feita da direita para esquerda. O eixo y representa as distâncias entre os grupos formados; o eixo $\mathrm{x}$ indica os grupos, em ordem decrescente de semelhança.

O primeiro conjunto (de maior semelhança) reúne cinco espécies, com dissimilaridade em torno de 0,4. A presença de raios heterogêneos, com mais de 3 células de largura em Mimosa pigra, aproxima a mesma de $M$. balduinii, bem como de $M$. adpressa e $M$. parvipinna, ambas Obstrigosae e muito semelhantes entre si; estas, por sua vez, distanciam-se de $M$. sparsa, que tem raios ainda mais largos, com 4 ou mais células de largura.

O segundo grupo reúne a maioria das espécies da seção Calothamnos, que apresentam raios altos (mais de $1400 \mu \mathrm{m}$ de altura), casos de Mimosa flocculosa, M. eriocarpa, M. pilulifera e $M$. daleoides; pertencente à mesma seção botânica, $M$. berroi agrupa-se a $M$. ramulosa (subsérie Obstrigosae), por ter raios de até 4 células de largura e 700-1400 $\mu \mathrm{m}$ de altura; a espécie restante da seção Calothamnos ( $M$. incana) aproxima-se de $M$. micropteris (série Myriophyllae), por apresentar raios ainda mais largos (mais de 4 células) e altos ( $>1400 \mu \mathrm{m})$. Da mesma seção Calothamnos, M. taimbensis agrupa-se a $M$. scabrella e a $M$. bimucronata, esta da seção Batocaulon e série Bimucronatae, devido aos raios homogêneos.

As seis espécies da série Stipellares apresentaram grande homogeneidade na análise de agrupamento devido aos poros em múltiplos racemiformes, bem como aos raios homogêneos e baixos (até $700 \mu \mathrm{m})$. Mimosa intricata e $M$. barnebiana, com caracteres anatômicos quase idênticos, compõem um subgrupo com pouca 
TABELA 2 - Distribuição das características anatômicas nas espécies em estudo.

\begin{tabular}{|c|c|c|c|c|c|c|c|c|c|c|c|c|c|c|}
\hline \multirow[b]{2}{*}{ Espécies } & \multicolumn{14}{|c|}{ Caracteres anatômicos } \\
\hline & - & 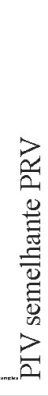 & 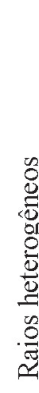 & 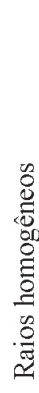 & 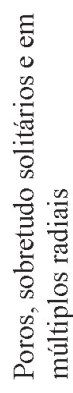 & 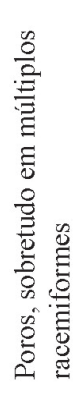 & 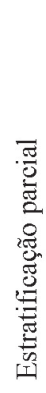 & 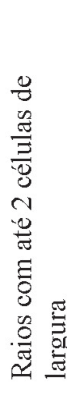 & 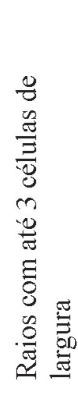 & 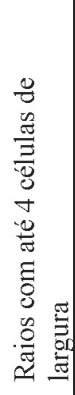 & 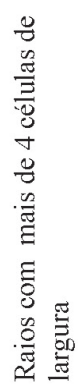 & 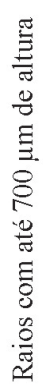 & 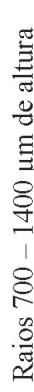 & 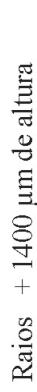 \\
\hline M. adpressa & 1 & 1 & 1 & 0 & 1 & 0 & 0 & 0 & 1 & 0 & 0 & 1 & 0 & 0 \\
\hline M. balduinii & 1 & 1 & 1 & 0 & 1 & 0 & 0 & 0 & 0 & 0 & 1 & 0 & 1 & 0 \\
\hline M. barnebiana & 1 & 1 & 0 & 1 & 0 & 1 & 0 & 0 & 1 & 0 & 0 & 1 & 0 & 0 \\
\hline M. berroi & 1 & 1 & 1 & 0 & 1 & 0 & 1 & 0 & 0 & 1 & 0 & 0 & 1 & 0 \\
\hline M. bifurca & 1 & 1 & 0 & 1 & 0 & 1 & 0 & 1 & 0 & 0 & 0 & 1 & 0 & 0 \\
\hline M. bimucronata & 1 & 1 & 0 & 1 & 1 & 0 & 0 & 0 & 0 & 1 & 0 & 1 & 0 & 0 \\
\hline M. cruenta & 1 & 1 & 1 & 0 & 0 & 1 & 0 & 1 & 0 & 0 & 0 & 1 & 0 & 0 \\
\hline M. daleioides & 1 & 1 & 1 & 0 & 1 & 0 & 1 & 0 & 0 & 1 & 0 & 0 & 0 & 1 \\
\hline M. eriocarpa & 1 & 1 & 1 & 0 & 1 & 0 & 0 & 0 & 0 & 1 & 0 & 0 & 0 & 1 \\
\hline M. flocculosa & 1 & 1 & 1 & 0 & 1 & 0 & 0 & 0 & 0 & 1 & 0 & 0 & 0 & 1 \\
\hline M. incana & 1 & 1 & 1 & 0 & 1 & 0 & 1 & 0 & 0 & 0 & 1 & 0 & 0 & 1 \\
\hline M. intricata & 1 & 1 & 0 & 1 & 0 & 1 & 0 & 0 & 1 & 0 & 0 & 1 & 0 & 0 \\
\hline M. micropteris & 1 & 1 & 1 & 0 & 1 & 0 & 0 & 0 & 0 & 0 & 1 & 0 & 0 & 1 \\
\hline M. parvipinna & 1 & 1 & 1 & 0 & 1 & 0 & 0 & 0 & 1 & 0 & 0 & 1 & 0 & 0 \\
\hline M. pigra & 1 & 1 & 1 & 0 & 1 & 0 & 0 & 0 & 1 & 0 & 0 & 0 & 1 & 0 \\
\hline M. pilulifera & 1 & 1 & 1 & 0 & 1 & 0 & 1 & 0 & 0 & 0 & 1 & 0 & 0 & 1 \\
\hline M. ramulosa & 1 & 1 & 1 & 0 & 1 & 0 & 0 & 0 & 0 & 1 & 0 & 0 & 1 & 0 \\
\hline M. scabrella & 1 & 1 & 0 & 1 & 1 & 0 & 1 & 0 & 0 & 1 & 0 & 0 & 0 & 1 \\
\hline M. sparsa & 1 & 1 & 1 & 0 & 1 & 0 & 0 & 0 & 0 & 1 & 0 & 1 & 0 & 0 \\
\hline M. taimbensis & 1 & 1 & 0 & 1 & 1 & 0 & 1 & 0 & 0 & 0 & 1 & 0 & 0 & 1 \\
\hline M. trachycarpa & 1 & 1 & 0 & 1 & 0 & 1 & 0 & 0 & 0 & 0 & 1 & 1 & 0 & 0 \\
\hline M. uraguensis & 1 & 1 & 0 & 1 & 0 & 1 & 0 & 0 & 0 & 1 & 0 & 1 & 0 & 0 \\
\hline
\end{tabular}

Fonte: autores.

Onde: $1=$ caráter presente. $0=$ caráter ausente. $\mathrm{PIV}=$ pontoação intervascular.

$\mathrm{PRV}=$ pontoação parênquimo-vascular. 
FIGURA 1 - Dendrograma de agrupamento das 22 espécies estudadas, utilizando-se a distância de Jaccard e o método UPGMA.

\section{Cluster Dendrogram}

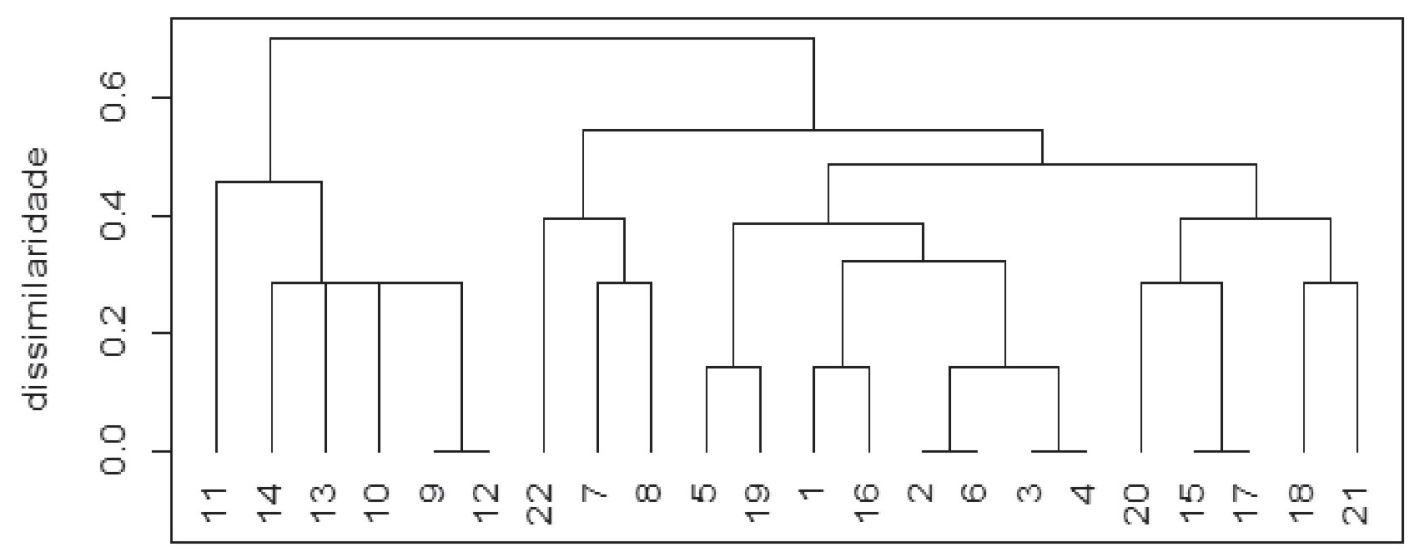

jaccard

hclust (*, "average")

Fonte: Autores.

Onde: 1, M. berroi; 2, M. daleoides; 3, M. eriocarpa; 4, M. flocculosa; 5, M. incana; 6, M. pilulifera; 7, $M$. scabrella; 8, M. taimbensis; 9, M. barnebiana; 10, M. bifurca; 11, M. cruenta; 12, M. intricata; 13, M. uraguensis; 14, M. tachycarpa; 15, M. parvipinna; 16, M. ramulosa; 17 , M. adpressa; 18, M. balduinii; 19 , M. micropteris; 20, M. sparsa; 21, M. pigra; 22, M. bimucronata. $R=0,85$;

dissimilaridade. A respeito das outras três espécies, separadas apenas pela largura de raios, Mimosa bifurca (até 2 células), M. uraguensis (até 4 células) e $M$. trachycarpa (mais que 4 células) compõem um subgrupo com índice de dissimilaridade ainda menor, em torno de 0,3. Única espécie com raios heterogêneos na série Stipellares, M. cruenta é a última agrupada e com índice de dissimilaridade 0,5.

\section{DISCUSSÃO}

A heterogeneidade anatômica do lenho em Mimosa L., reconhecida, inicialmente, por Cozzo (1949, 1951) e Marchiori (1980), é plenamente reafirmada no presente estudo, visto que o grupo de espécies investigadas reúne qua- se todos os caracteres anatômicos atribuídos tanto ao atual clado Mimosoideae, como à subfamília Caesalpinioideae sensu LPWG (2017).

Em sua abordagem evolutiva do lenho em Leguminosae, Bareta-Kuipers (1981) reconheceu que as Caesalpinioideae constituem a base da família e que, a partir delas, as Mimosoideae evoluíram no tocante aos raios e as Papilionoideae em direção à estratificação completa, ou seja, de elementos vasculares, raios, parênquima axial e fibras. A autora também afirma que a estrutura do lenho na tribo Caesalpinieae assemelha-se ao observado em Mimosoideae, e que a estratificação de raios unisseriados em Cercideae distinguia essa tribo do restante das Caesalpiniodeae. 
A recente contribuição do Legume Phylogeny Working Group (LPWG, 2017) à filogenia de Fabaceae, que acabou por incorporar Mimosoideae em Caesalpinioideae após segregação de alguns grupos desta subfamília (Cercideae, entre outros), é reforçada no presente estudo, posto que a variabilidade anatômica em Mimosa L. corresponde ao atribuído a toda a subfamília: raios homogêneos e heterogêneos; poros tanto solitários e em curtos múltiplos, como em múltiplos racemiformes, por vezes tendentes a arranjo diagonal; parênquima axial com séries de duas ou mais células; e presença (ou não) de parênquima axial estratificado.

Os caracteres quantitativos que comumente servem na diferenciação de espécies, tais como abundância de poros (poros $/ \mathrm{mm}^{2}$ ), largura de raios (tanto em $\mu \mathrm{m}$ como em número de células), altura de raios ( $\mu \mathrm{m})$, e comprimento de fibras $(\mu \mathrm{m})$, carecem de valor filogenético. $\mathrm{O}$ diâmetro de poros, apesar da estreita correlação ecofisiológica (Carlquist, 1975; Dickison, 1975; Metcalfe \& Chalk, 1979), também não se aplica à taxonomia e filogenia.

$\mathrm{Na}$ literatura é amplamente documentada a especialização dos raios homogêneos em relação a heterogêneos (Carlquist, 1975; Dickison, 1975; Metcalfe \& Chalk, 1979), explicando-se o dilema pela adequação das células procumbentes de raios homogêneos à condução de seiva elaborada, ligando o floema secundário ao interior da madeira, onde se dá o armazenamento, sobretudo em células de parênquima axial. Os raios heterogêneos, em contraste, são menos especializados, visto que as células quadradas e eretas, pouco eficientes na condução e geralmente confinadas às margens, são mais adequadas ao armazenamento de substâncias de reserva, funcionando à semelhança do parênquima axial. Neste sentido, a ocorrência de raios homogêneos e heterogêneos em Mimosa L. é caráter primitivo que sinaliza, para o gênero em estudo, um posicionamento mais próximo à base evolutiva de Caesalpinioideae.

Além de caráter evoluído, a presença de raios homogêneos em Mimosa barnebiana, M. bi- furca, $M$. cruenta, $M$. intricata, $M$. trachycarpa e M. uraguensis, todas da seção Batocaulon DC. (ou série Stipellares Benth.), também apresenta valor diagnóstico para esse grupo, tendo sido observado, fora do mesmo, apenas em Mimosa bimucronata (seção Batocaulon e série Bimucronatae), bem como em Mimosa scabrella e Mimosa taimbensis, da seção Calothamnos.

Sob o ponto de vista taxonômico, a estratificação do parênquima axial em todas as espécies investigadas da série Stipellares demonstra o valor diagnóstico do caráter para a referida seção, até mesmo número expressivo de espécies investigadas: seis, das dezenove reconhecidas por Barneby (1991).

Tendo em vista que a estratificação é caráter especializado (Carlquist, 1975; Dickison, 1975; Jane, 1962; Metcalfe \& Chalk, 1979; Panshin $\&$ De Zeeuw, 1970), fica reforçada a interpretação da série Stipellares Benth. como grupo infragenérico evoluído.

No presente estudo, a predominância de poros em múltiplos racemiformes - e com tendência a arranjo dendrítico - foi observada apenas nas seis espécies da série Stipellares, fato que demonstra a importância taxonômica dos mesmos para o referido grupo (Machado et al., 2016). Em anatomia ecológica, esse caráter, geralmente associado ao hábito arbustivo e ambientes secos (Santos \& Marchiori, 2010), é reconhecido desde o trabalho pioneiro de Carlquist (1975). Em Mimosa L., entretanto, não se verifica essa associação, uma vez que os vasos nas espécies investigadas da subsérie Obstrigosae (M. adpressa, M. parvipinna e M. ramulosa) não apresentam esse arranjo, mesmo sendo plantas ainda menores na natureza e de ambientes mais xerófilos, do que as espécies investigadas da série Stipellares.

A seção Calothamnos, representada por oito espécies no presente estudo, distingue-se pelos raios superiores a $1400 \mu \mathrm{m}$, casos de Mimosa daleoides, M. eriocarpa. M. flocculosa, $M$. incana, $M$. pilulifera, $M$. scabrella e $M$. taimbensis, aspecto compartilhado, tão somente, por Mimosa micropteris (série 
Myriophyllae). Apenas Mimosa berroi, que é um subarbusto, apresenta raios mais baixos

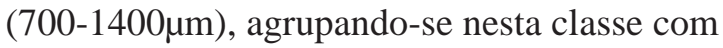
M. ramulosa (Série Mimosa, Subsérie Obstrigosae).

Representada por três espécies (Mimosa adpressa, M. parvipinna e $M$. ramulosa), a subsérie Obstrigosae (Benth.) Barneby é de mais difícil reconhecimento anatômico, comparada à Série Stipellares e Seção Calothamnos. As duas primeiras espécies apresentam raios

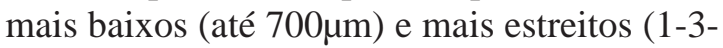
seriados); $M$. ramulosa distingue-se pelos raios ligeiramente mais altos e com até 4 células de largura. Tais caracteres, obviamente, são de escasso valor taxonômico, não custa frisar.

Com relação às demais espécies investigadas, qualquer observação de caráter taxonômico ou filogenético esbarra na reduzida amostragem. É o caso da série Bimucronatae (M. bimucronata), da série Habbasia (M. pigra), da série Myriophyllae (M. balduinii e $M$. micropteris) e da subsérie Sparsae (M. sparsa), que foram investigadas por apenas uma ou duas espécies, devido à escassa representatividade na flora regional.

\section{CONCLUSÕES}

A análise de agrupamento de caracteres anatômicos do lenho de vinte e duas espécies de Mimosa L., nativas no Sul do Brasil, confirma a heterogeneidade estrutural referida na literatura e permite as seguintes conclusões:

- A ocorrência de caracteres primitivos e evoluídos na estrutura do lenho recomenda um posicionamento basal para o referido gênero, no clado Mimosoideae e subfamília Caesalpinioideae.

- A série Stipellares Benth. compõe um grupo distinto sob os pontos de vista taxonômico e filogenético, sobretudo pelos raios homogêneos e poros em múltiplos racemiformes, com arranjo tendente a dendrítico.

- Apesar do número expressivo de espécies investigadas, tanto a seção Calothamnos Barneby como a subsérie Obstrigosae (Benth.)
Barneby não dispõem de caracteres de valor taxonômico ou filogenético reconhecidos na literatura, sendo distinguidas com base em aspectos meramente quantitativos.

- O reduzido número de espécies investigadas das séries Bimucronatae Barneby, Habbasia (DC.) Barneby, Myriophyllae Benth. e da Subsérie Sparsae Barneby, não permite inferências taxonômicas e filogenéticas.

\section{LITERATURA CITADA}

BARETA-KUIPERS, T. Wood anatomy of Leguminosae: its relevance to Taxonomy. In: POLHILL, R.M.; RAVEN, P.H. Advances in Legume Systematics. 1981. p. 677-715.

BARNEBY, R.C. Sensitivae Censitae: a description of the genus Mimosa Linnaeus (Mimosaceae) in the New World. Memoirs of the New York Botanical Garden, v. 65, p. 1-835, 1991.

BENTHAM, G. Revision of the suborder Mimoseae. Transact Linnean Society of London, v. 30, p. 335-664, 1875.

BURKART, A. Leguminosas Mimosoideas. In: REITZ, P.R. Flora Ilustrada Catarinense. Itajaí: Herbário "Barbosa Rodrigues", 1979. 299 p.

CARNIELETTO, C.; MARCHIORI, J.N.C. Anatomia da madeira de Mimosa eriocarpa Benth. Ciência Florestal, Santa Maria, v. 3, n. 1, p. 107-120, 1993.

CARLQUIST, S. Ecological strategies of xylem evolution. Berkeley: University of California Press, 1975. 259p.

COZZO, D. Estructura leñosa estratificada no registrada en géneros de Leguminosas argentinas. Lilloa, Tucuman, v. 16, p. 63-95, 1949.

COZZO, D. Anatomía del leño secundario de las Leguminosas Mimosoideas y Cesalpinioideas argentinas silvestres y cultivadas. Revista del Instituto Nacional de Investigación de las Ciencias Naturales, Ciencias Botánicas, Buenos Aires, v. 2, n. 2, p. 63-290, 1951.

DICKISON, W.C. The bases of Angiosperm Phylogeny: Vegetative Anatomy. Annals of the Missouri Botanical Garden, Saint Louis, n. 62, p. 590-620, 1975.

JANE, F.W. The structure of wood. London: Adam \& Charles Black, 1962. 427p.

LPWG - The Legume Phylogeny Working Group. A 
new subfamily classification of the Leguminosae based on a taxonomically comprehensive Phylogeny. Taxon, v. 66, n. 1, p. 44-77, 2017.

MACCARI, A.; MARCHIORI, J.N.C. Estudo anatômico do xilema secundário de Mimosa sparsa Benth. Ciência Florestal, Santa Maria, v. 4, n. 1, p. 145-155, 1994.

MACHADO, P.F.S.; MARCHIORI, J.N.C. Anatomia do lenho de duas espécies sul-brasileiras do gênero Mimosa L. e subsérie Obstrigosae (Benth.) Barneby. Balduinia, Santa Maria, n. 52, p. 22-29, 2016a.

MACHADO, P.F.S.; MARCHIORI, J.N.C. Anatomia do lenho de Mimosa pigra Benth. Balduinia, Santa Maria, n. 55, p. 17-22, 2016 b.

MACHADO, P.F.S.; MARCHIORI, J.N.C. Anatomia dos lenhos de Mimosa intricata Benth. e Mimosa taimbensis Burkart (Fabaceae). Balduinia, Santa Maria, n. 57, p. 19-29, 2017a.

MACHADO, P.F.S.; MARCHIORI, J.N.C. Anatomia da madeira de Mimosa balduinii Burkart. Balduinia, Santa Maria, n. 58, p. 25-32, 2017b.

MACHADO, P.F.S.; MARCHIORI, J.N.C. Anatomia do lenho de Mimosa adpressa Hook. \& Arn. (Fabaceae; Caesalpinioideae). Balduinia, Santa Maria, n. 60, p. 29-34, 2017c.

MACHADO, P.F.S.; MARCHIORI, J.N.C.; BALDIN, T. Anatomia da madeira de duas espécies de Mimosa da série Stipellares Benth. Balduinia, Santa Maria, n. 54, p. 1-10, 2016.

MARCHIORI, J.N.C. Estudo anatômico do xilema secundário e da casca de algumas espécies dos gêneros Acacia e Mimosa, nativas no estado do Rio Grande do Sul. 1980. 185p. Dissertação (Mestrado em Engenharia Florestal) Universidade Federal do Paraná, Curitiba, PR, 1980.

MARCHIORI, J.N.C. A estrutura do xilema secundário de Mimosa daleoides Benth. (Leguminosae Mimosoideae). Ciência e Natura, Santa Maria, n. 4, p. 107-113, 1982.

MARCHIORI, J.N.C. Anatomia da madeira e casca do maricá, Mimosa bimucronata (DC.) Kuntze. Ciência Florestal, Santa Maria, v. 3, n. 1, p. 85106, 1993.

MARCHIORI, J.N.C. Anatomia da madeira de Mimosa cruenta Benth. (Leguminosae Mimosoideae). Ciência e Natura, Santa Maria, n. 7, p. 73-81, 1885.
MARCHIORI, J.N.C. Anatomia da madeira e casca da bracatinga, Mimosa scabrella Benth. Ciência e Natura, Santa Maria, n. 17, p. 115-132, 1995.

MARCHIORI, J.N.C. Anatomia do xilema secundário de Mimosa berroi Burk. Ciência e Natura, Santa Maria, n. 18, p. 117-129, 1996a.

MARCHIORI, J.N.C. Anatomia do xilema secundário de Mimosa uraguensis Hook. \& Arn. Ciência e Natura, Santa Maria, v. 18, p. 103115, 1996b.

MARCHIORI, J.N.C. Anatomia do xilema secundário de Mimosa incana (Spreng.) Benth. Ciência Florestal, Santa Maria, v. 6, n. 1, p. 5363, 1996c.

MARCHIORI, J.N.C.; MUÑIZ, G.I.B. de. Estudo anatômico do xilema secundário de Mimosa pilulifera Benth. Ciência Florestal, Santa Maria, v. 7, n. 1, p. 65-75, 1997a.

MARCHIORI, J.N.C.; MUÑIZ, G.I.B. de. Estudo anatômico do xilema secundário de Mimosa trachycarpa Benth. Ciência Rural, Santa Maria, v. 27, n. 2, p. 223-228, 1997 b.

METCALFE, C.R.; CHALK, L. Anatomy of Dicotyledons. Oxford: Clarendon Press, 1972. $1500 \mathrm{p}$.

METCALFE, C.R.; CHALK, L. Anatomy of Dicotyledons. Oxford: Clarendon Press, 1979. $2^{a}$ ed. v. 1.276 p.

OKSANEN, J.; BLANCHET, F.G.; KINDT, R.; LEGENDRE, P.; O'HARA, R.B.; SIMPSON, G.L.; STEVENS, M.H.H.; WAGNER, H. vegan: community ecology package. Version 2.0-6. 2012. Avaiable from: <http://vegan.r-forge.rproject.org/>.

PANSHIN, A.J.; DE ZEEUW, C. Textbook of Wood Technology. New York: McGraw-Hill, 1970. v. $1.705 \mathrm{p}$.

PEREIRA, A.C. da S.; SIEGLOCH, A.M.; MARCHIORI, J.N.C. Anatomia do lenho de Mimosa flocculosa Burkart. Balduinia, Santa Maria, n. 43, p. 29-33, 2013.

RAMBO, B. Leguminosae Riograndenses. Pesquisas, Botânica, São Leopoldo, n. 23, p. 1166, 1966.

RECORD, S.J.; HESS, R.W. Timbers of the New World. New Haven: Yale University Press, 1949. 640p.

SANTOS, S.R. dos; MARCHIORI, J.N.C.; CANTODOROW, T.S. do. Identificação botânica e 
anatômica da madeira de Discaria americana

Gillies \& Hooker. Balduinia, Santa Maria, n, 10,

p., 11-20, 2007.
SIEGLOCH, A.M.; MARCHIORI, J.N.C; SANTOS, S.R. dos. Anatomia do lenho de Mimosa micropteris Benth. Balduinia, Santa Maria, n. 40, p. 18-22, 2013. 